\title{
ROTOR-BLADE VIBRATION CONTROL USING A PERIODIC LQR CONTROLLER
}

\author{
C. S. Jakobsen*, J. F. CAmino ${ }^{\dagger}$, I. F. SAntos* \\ * Department of Mechanical Engineering \\ Technical University of Denmark \\ DK-2800, Kgs. Lyngby, Denmark \\ $\dagger$ School of Mechanical Engineering \\ University of Campinas \\ 13083-860, Campinas, SP, Brazil
}

Emails: csjmail@gmail.com, camino@fem.unicamp.br, ifs@mek.dtu.dk

\begin{abstract}
This paper presents an active control strategy based on the periodic time varying linear quadratic regulator problem to attenuate the vibration of a coupled rotor-blade system via shaft actuation. For this system, a periodic modal decomposition method is applied to decouple part of the system dynamics, which ultimately provides a reduced order model. Then, a periodic LQR controller obtained as the solution of a periodic Riccati differential equation is designed. For comparison purpose, three others controllers are also designed. A proportional derivative controller, an LQR controller based on a single LTI model, and an LQR controller based on multiple LTI models. Numerical simulations show the superior performance of the proposed technique.
\end{abstract}

Keywords - Rotor dynamics, vibration control, periodic systems, LQR controller.

\section{Introduction}

Although active control of vibrations for flexible structures has been extensively studied for several decades (Balas, 1978; Firoozian and Stanway, 1988; Khulief, 2001; Baz and Ro, 2001) its application to rotor-blade systems using shaft-based actuation is still an open area of research. Most of the rotor-blade control design techniques assume, by neglecting the rotor lateral motion and by considering that the rotor operates at constant angular velocity, that the system is linear and time invariant. However, in a more realistic application, this is not the case and the system becomes periodic, requiring more elaborate strategies.

Control design techniques that takes into account the fact that the system is periodic and time varying have been reported during the last two decades (Arcara et al., 2000; Sinha and Joseph, 1994a; Szász and Flowers, 2001). It was shown in Szász and Flowers (2001) that the rotor and the blade vibrations can be controlled by a shaft based actuation if the rotor blades are deliberately mistuned. In this work, the presence of parametric vibrations due to the coupling among the rotor and the blades were not considered. Such effects were analyzed in Christensen (2004) by considering the modal controllability and observability of a coupled rotor-blade system that shows significant vibration coupling among the rotor and the blades. In Christensen and Santos (2005a), an experimental contribution is given that shows the feasibility of a periodic modal control strategy.

The main contribution of our paper is to provide a control strategy based on the periodic time varying linear quadratic regulator (LQR) problem to attenuate the vibration of coupled rotor-blade systems via shaft actuation, in which the rotor lat- eral motion and the blade flexible motion are coupled. An analytical periodic time varying model that describes the dynamics of the rotor-blade system is developed. The rotor angular motion and the gyroscopic effect are neglected for simplicity, and the blades are modeled as flexible Bernoulli beams. For this system, a periodic modal decomposition ( $\mathrm{Xu}$ and Gasch, 1995) is applied to transform the system dynamics matrix into a decoupled time invariant matrix. Afterward, the system order is reduced. For this reduced order model, a periodic LQR controller, obtained as the solution of a periodic Riccati differential equation, is designed. For comparison purpose, three others controllers (based on Christensen and Santos (2005b)) are used: a proportional derivative controller; an LQR controller based on a single LTI model; and an LQR controller based on multiple LTI models. Numerical simulations show the superior performance of the proposed periodic controller, which is capable to cope with the time periodicity of the system and to efficiently suppress the main vibration modes. Moreover, the proposed periodic LQR controller is able to guarantee stability of the closed-loop rotor-blade system.

\section{Rotor-blade system}

The mechanical system is a two-dimensional fourbladed rotor with tip masses, rotating in a suspended hub, which can be actuated in two perpendicular radial directions as shown in Fig. 1. The hub motion is described by the $\left(x_{h}, y_{h}\right)$ position in the fixed $(x, y)$-coordinate system. The deflection of an arbitrary point on the $i$-th blade is described by the $\left(x_{i}, y_{i}\right)$ position in the moving $\left(x_{b_{i}}, y_{b_{i}}\right)$-coordinate system. The angular position 
of blade 1 is given by $\theta(t)$. For a detailed explanation see Christensen and Santos (2005b).

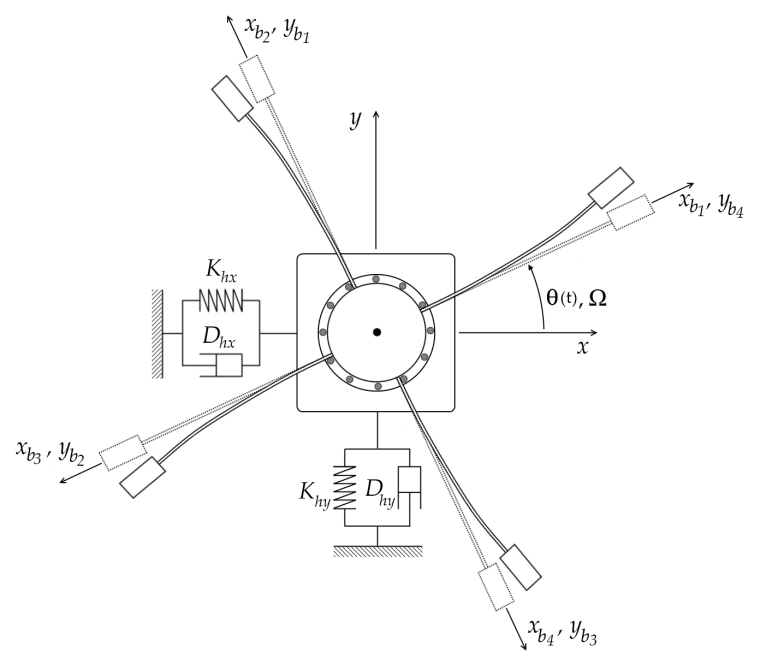

Figure 1: Two-dimensional four-bladed rotorblade system.

The analytical model was derived using the Lagrangian formalism. The resulting model is a time varying system that depends on the rotorposition $\theta(t)$ and on the rotational speed $\Omega$. By rotating at constant speed, this model can be rewritten in a periodic time varying form, with period $T=2 \pi / \Omega$, given by

$$
M(t) \ddot{z}(t)+D(t) \dot{z}(t)+K(t) z(t)=p(t)+Q u(t)
$$

where the periodic matrices $M(t), D(t)$ and $K(t)$ represent, respectively, the mass, damping and stiffness of the system, $Q$ is an input matrix, $u(t)$ is the control force that acts on the hub, and $p(t)$ represents internal periodic conservative forces originating from the hub unbalance and the unbalance due to the mistuned blades. The state of the system is $z(t)=\left\{x_{h} ; y_{h} ; q_{1} ; q_{2} ; q_{3} ; q_{4}\right\}$, where the vector $q_{i}(t)=\left\{q_{i, 1}(t) ; \ldots ; q_{i, m}(t)\right\}$ represents the generalized time-dependent coordinate associated with the spatial mode shape $\varphi_{i}\left(x_{i}\right)$ of the $i$-th blade, i.e., the transverse deformation of the blade was discretized using the following expansion $y_{i}\left(x_{i}, t\right)=\sum_{j=1}^{m} \varphi_{i, j}\left(x_{i}\right) q_{i, j}(t)=$ $\varphi_{i}\left(x_{i}\right) q_{i}(t)$, where $m$ defines the number of blade bending modes. The total degrees of freedom is $N=2+4 m$.

The physical output of the system, at the employed sensors, is given by

$$
y(t)=C_{h} z(t)
$$

This matrix $C_{h}$ transforms the $z$ coordinate into the physical form $y(t)=\left\{x_{h} ; y_{h} ; y_{1} ; y_{2} ; y_{3} ; y_{4}\right\}$, which is defined in the system coordinate. The hub based degrees of freedom $x_{h}$ e $y_{h}$ are already in physical coordinates, but the blade tip deflection $y_{i}$ is a combination of all the $m$ modes of the blade.
By defining the state vector

$$
x(t)=\left\{\begin{array}{l}
z(t) \\
\dot{z}(t)
\end{array}\right\}
$$

equation (1) can be represented in the following periodic time varying state-space form

$$
\begin{aligned}
\dot{x}(t) & =A(t) x(t)+B(t) u(t)+f(t) \\
y(t) & =C x(t)
\end{aligned}
$$

with

$$
\begin{aligned}
& A(t)=\left[\begin{array}{cc}
0 & I \\
-M(t)^{-1} K(t) & -M(t)^{-1} D(t)
\end{array}\right], \\
& B(t)=\left[\begin{array}{c}
0 \\
-M(t)^{-1} Q
\end{array}\right], \quad f(t)=\left\{\begin{array}{c}
0 \\
-M(t)^{-1} p(t)
\end{array}\right\}, \\
& C=\left[\begin{array}{ll}
C_{h} & 0
\end{array}\right]
\end{aligned}
$$

\subsection{Periodic modal decomposition}

The periodic equation (2) can be partially decoupled using an appropriate transformation matrix (Sinha and Joseph, 1994b; Xu and Gasch, 1995; Christensen and Santos, 2005b). By introducing the Lyapunov-Floquet transformation $x(t)=R(t) \xi(t)\left(\right.$ and $\left.\xi(t)=L^{T}(t) x(t)\right)$, where $\xi(t)$ is a vector of modal states and $R(t)$ and $L(t)$ are the right- and left-side modal transformation matrices, one obtains the following modal equation

$$
\begin{aligned}
\dot{\xi}(t) & =\mathcal{A} \xi(t)+\mathcal{B}(t) u(t)+\mathfrak{f}(t) \\
y(t) & =\mathcal{C}(t) \xi(t)
\end{aligned}
$$

with

$$
\begin{aligned}
\mathcal{A} & =\left[L^{T}(t) A(t) R(t)-L^{T}(t) \dot{R}(t)\right], \\
\mathcal{B}(t) & =L^{T}(t) B(t), \quad \mathfrak{f}(t)=L^{T}(t) f(t), \\
\mathcal{C}(t) & =C R(t)
\end{aligned}
$$

The periodic matrices $R(t)$ and $L(t)$ are determined by solving the associated time-dependent eigenvalue problem. Note that the system matrix $A(t)$ was transformed into a time invariant diagonal modal matrix $\mathcal{A}$, however, all the others matrices still remain time varying.

\subsection{Model order reduction}

Only the first bending mode, being the far most significant (Christensen and Santos, 2005b) is considered for control design. Therefore, the modal model (4) is reduced to a six degrees of freedom system, containing only the two modes related to the hub motion and the first bending mode of the four blades, giving a system of dimension $N=12$.

Since (4) can contain complex entries, it is also appropriate to partition the state coordinate into real- and imaginary parts giving the following model (of twice the size) with real entries:

$$
\begin{aligned}
\dot{\tilde{\xi}}(t) & =\tilde{\mathcal{A}} \tilde{\xi}(t)+\tilde{\mathcal{B}}(t) u(t)+\tilde{\mathfrak{f}}(t) \\
y(t) & =\tilde{\mathcal{C}}(t) \tilde{\xi}(t)
\end{aligned}
$$




\section{Controller design}

It was shown in Szász and Flowers (2001) and Christensen and Santos (2005b) that the vibration of the two-dimensional four-bladed rotor-blade system can be attenuated using control forces acting on the hub. However, classical control design procedures can not be directly applied since the equation of motion of the system is periodic time varying. Moreover, system stability cannot be inferred from the location of the closed-loop eigenvalues as done for linear time invariant systems. Recall that the periodic modal decomposition, applied in the previous section, only transformed the periodic time varying system matrix $A(t)$ into a constant modal matrix $\mathcal{A}$, however the modal matrices $\mathcal{B}(t)$ and $\mathcal{C}(t)$ are still time dependent.

To cope with the periodic time varying nature of the problem, a periodic LQR design is proposed in Sec. 3.1. This methodology is able to significantly improve the performance and robustness of the rotor-blade system. This technique is compared to three different controllers. A proportional derivative controller taken from Christensen and Santos (2006) and described in Sec. 3.5. A single frozen model method taken from Sinha and Joseph (1994b) and Christensen and Santos (2005b). This method, presented in Sec. 3.3, applies a time invariant LQR design to a properly chosen LTI model. As an extension of this method, a multiple frozen model method is proposed in Sec. 3.4, which computes LQR controllers for multiple LTI models obtained trough a gridding of the input matrix $\mathcal{B}(t)$.

\subsection{Linear quadratic regulator design}

The optimal full state feedback gain is obtained as a solution of an algebraic Riccati equation (ARE). The LQR problem can also be solved efficiently using linear matrix inequalities (Willems and Mitter, 1971; Boyd et al., 1994). For linear time varying systems, the optimal LQR gain is obtained as a solution of a differential Riccati equation (Moore and Anderson, 1968a; Moore and Anderson, 1968b; Athans, 1971; Anderson and Moore, 1990).

Consider the following system

$$
\dot{x}(t)=A(t) x(t)+B(t) u(t)
$$

The central element of the LQR problem is the design of a full state feedback law

$$
u(t)=G(t) x(t)
$$

that minimizes the quadratic cost function

$$
J=\int_{0}^{\infty}\left(x^{T}(t) W_{Q}(t) x(t)+u^{T}(t) W_{R}(t) u(t)\right) d t
$$

with symmetric matrices $W_{R}(t)>0$ and $W_{Q}(t) \geq$ 0 . The matrix $W_{Q}$ penalizes the system state meanwhile the matrix $W_{R}$ penalizes the control input. The optimal full state feedback gain, which guarantees that the closed-loop system is asymptotically stable, is given by

$$
G(t)=-W_{R}(t)^{-1} B(t)^{T} X(t)
$$

where the matrix $X(t)$ is the solution of the following differential Riccati equation (DRE):

$$
\begin{array}{r}
-\dot{X}(t)=A(t)^{T} X(t)+X(t) A(t)+W_{Q}(t) \\
-X(t) B(t) W_{R}^{-1}(t) B^{T}(t) X(t)
\end{array}
$$

Once this DRE is solved, the optimal feedback gain $G(t)$ is readily obtained.

\subsection{Solving the PRDE}

When the system matrices in the LQR problem are periodic, three methods are available to obtain a suitable matrix solution $X(t)$ of the periodic differential Riccati equation (6), namely the one-shot method, suggested in Hench and Laub (1994) and evaluated in Johansson et al. (2007), the multishot method, suggested in Varga (2005) and also evaluated in Johansson et al. (2007), and the LMI approach suggested in Gusev et al. (2007).

The one-shot technique is by far the most simple method to implement. The method performs well (for short periods $T$ ) on small scale systems. The multi-shot method has been designed to overcome some of the limitations of the oneshot method, however, to implement this method, it is necessary to compute and reorder a periodic real Schur decomposition which is also a cumbersome task for large scale systems.

A completely different approach, posed in the linear matrix inequality (LMI) framework, is presented in Gusev et al. (2007). The method transforms the time varying periodic matrices into time invariant form via Fourier expansion and replace the DRE by an a matrix inequality. Thereby, an LMI optimization problem can be set up. The LMI problem has been implemented using the Yalmip toolbox for Matlab (Löfberg, 2004) and solved using the SeDuMi solver (Sturm, 1999). Since this method is computationally demanding, the reduced non-complex modal system given by (5) was used for the LQR design from Sec. 3.1. Once the full state feedback gain is computed, it can easily be transformed to the original form (2).

\subsection{Single frozen model design}

The main idea of the single frozen model method (Sinha and Joseph, 1994b; Szász and Flowers, 2001; Christensen and Santos, 2005b) is to apply, to the model (5), a standard LTI control design technique computed using a frozen control pair $\tilde{\mathcal{A}}$ and $\tilde{\mathcal{B}}_{0}$. In Szász and Flowers (2001), the matrix 
$\tilde{\mathcal{B}}_{0}$ has been chosen as the identity matrix, however, in Christensen and Santos (2005b), it was chosen as $\tilde{\mathcal{B}}_{0}=\tilde{\mathcal{B}}\left(t_{0}\right)$ for an appropriate instant of time $t_{0}$.

Now, the classical time invariant LQR procedure can be applied to the LTI modal system

$$
\dot{\tilde{\xi}}(t)=\tilde{\mathcal{A}} \tilde{\xi}(t)+\tilde{\mathcal{B}}_{0} u(t)
$$

to provide the control law

$$
u(t)=\tilde{\mathfrak{G}}_{0} \tilde{\xi}(t)
$$

that minimize the cost function

$$
J=\int_{0}^{\infty}\left(\tilde{\xi}^{T}(t) W_{Q}(t) \tilde{\xi}(t)+u^{T}(t) W_{R}(t) u(t)\right) d t
$$

Once the constant full state feedback gain $\tilde{\mathfrak{G}}_{0}$ is obtained, it can be converted (as described in Sinha and Joseph (1994b) and Christensen and Santos (2005b)) into a periodic time varying gain by solving for $\mathfrak{G}(t)$ the following equation

$$
\tilde{\mathcal{B}}(t) \tilde{\mathfrak{G}}(t) \tilde{\xi}(t)=\tilde{\mathcal{B}}_{0} \tilde{\mathfrak{G}}_{0} \tilde{\xi}(t)
$$

Since $\tilde{\mathcal{B}}(t)$ is a nonsquare matrix, the time varying gain $\tilde{\mathfrak{G}}(t)$ is obtained via the Moore-Penrose pseudoinverse as

$$
\tilde{\mathfrak{G}}(t)=\left(\tilde{\mathcal{B}}^{H}(t) \tilde{\mathcal{B}}(t)\right)^{-1} \tilde{\mathcal{B}}^{H}(t) \tilde{\mathcal{B}}_{0} \tilde{\mathfrak{G}}_{0}
$$

It should be emphasized that this time varying gain does not guarantee optimality nor stability for the closed-loop time varying system.

\subsection{Multiple frozen models design}

The multiple frozen models method (MFM) is similar to the single frozen model method (SFM), presented in Sec. 3.3, in the way that the constant state feedback LQR gain is calculated. However, instead of trying to transform the constant gain matrix $\tilde{\mathfrak{G}}_{0}$ into a time varying gain $\tilde{\mathfrak{G}}(t)$ using the pseudoinverse, the MFM method distinguishes itself by computing multiple constant LQR gains $\tilde{\mathfrak{G}}_{i}$ for the pair $(\tilde{\mathcal{A}}, \tilde{\mathcal{B}}(t))$, calculated at suitable discretization of the period $T=2 \pi / \Omega$, and then generate the time varying gain $\tilde{\mathfrak{G}}(t)$ by switching amongst the designed constant gains $\tilde{\mathfrak{G}}_{i}$.

\subsection{Proportional derivative design}

The time invariant proportional derivative (PD) controller (as proposed in Christensen and Santos (2006)) is based on the feedback of the hub's position $x_{h}$ and velocity $y_{h}$. This control law is given by

$$
\begin{aligned}
& u(t)=-G_{1} y(t)-G_{2} \dot{y}(t) \\
& y(t)=\bar{C}_{h} z(t)
\end{aligned}
$$

For this application, the sensors and actuators are collated, thus the output matrix $\bar{C}_{h}$ is the transpose of the input matrix $Q$. By inserting the control law and the relation $\bar{C}_{h}=Q^{T}$ into the general equation of motion (1), one gets

$$
\begin{aligned}
M(t) \ddot{z}(t) & +D(t) \dot{z}(t)+K(t) z(t)= \\
& -Q\left(G_{1} Q^{T} z(t)+G_{2} Q^{T} \dot{z}(t)\right)+p(t)
\end{aligned}
$$

Equivalently

$$
\begin{aligned}
M(t) \ddot{z}(t)+\left(D(t)+Q G_{2} Q^{T}\right) \dot{z}(t) & \\
& +\left(K(t)+Q G_{1} Q^{T}\right) z(t)=p(t)
\end{aligned}
$$

After an iterative procedure (see (Christensen and Santos, 2006)), the gains $G_{1}$ and $G_{2}$ have been chosen as the following diagonal matrices:

$$
G_{1}=\left[\begin{array}{cc}
10^{4} & 0 \\
0 & 10^{4}
\end{array}\right], \quad G_{2}=\left[\begin{array}{cc}
50 & 0 \\
0 & 50
\end{array}\right]
$$

\subsection{Feedforward design}

The controllers developed in the previous sections did not include the known internal conservative forces $f(t) \equiv f(\theta, \Omega)$ due to unbalances. However, since the angular velocity $\Omega$ and the rotor position $\theta$ are known, it is possible to cancel out these forces using a feedforward compensator.

The control input $u(t)$ is split into two separate contributions $u(t)=u_{c}(t)+u_{f}(t)$, where $u_{c}(t)$ is the feedback control law, designed using the techniques presented in the previous sections, and $u_{f}(t)$ is the feedforward control law. From the equation of motion (2), it is seen that in order to cancel out the unbalance forces $f(t)$ the following equation must be fulfilled

$$
B(t) u_{f}(t)=-f(t)
$$

Since the matrix $B(t)$ is nonsquare, solving the above equation for $u_{f}(t)$ is not always possible. However, one or more solutions exist if

$$
f(t)=B(t) B^{\dagger}(t) f(t)
$$

holds, where $B^{\dagger}(t)$ is the Moore-Penrose pseudoinverse defined as $B^{\dagger}(t)=\left(B^{H}(t) B(t)\right)^{-1} B^{H}(t)$. Moreover, if the matrix $B(t)$ has full column rank (which is the case) then the solution is unique. A direct calculation, using the structure given in (3), shows that Eq. 8 is equivalent to

$$
M^{-1}(t) p(t)=V\left(V^{H} V\right)^{-1} M^{-1}(t) p(t)
$$

with $V=M^{-1}(t) Q$. Now, if the internal conservative force $p(t)$ has the form $p(t)=Q g(t)$, for some $g(t)$, then, the above equation reduces to

$$
V g(t)=V V^{\dagger} V g(t)=V g(t)
$$

Therefore, Eq. (8) is always satisfied whenever $p(t)$ has the form $p(t)=\left\{p_{1}(t) ; p_{2}(t) ; 0 ; \ldots ; 0\right\}$, which, 
in fact, is the case, as the unbalance only affects the hub's motions. Since the statement (8) holds, the feedforward law can be given by

$$
u_{f}(t)=-\left(B^{H}(t) B(t)\right)^{-1} B^{H}(t) f(t)
$$

\section{Numerical results}

The initial condition of the system has been chosen as $z(0)=\{1 ; 1 ; 1 ; 1 ;-1 ;-1\}[\mathrm{mm}]$, meaning that the hub is initially deflected one $\mathrm{mm}$ in both $(x, y)$-direction and the first mode of the blades are equally deflected one $\mathrm{mm}$. The reason for the negative sign of blade 3 and 4 is to make them move in phase with the opposite blade instead of completely out of phase, which would effectively cancel out the blade-hub coupling. The angular velocity, for these comparisons, will be set at $\Omega=300 \mathrm{rpm}$. The impact of the unbalance force $p(t)$ is only included in Sec. 4.5 , where the effect of the feedforward compensator is considered.

\subsection{Proportional derivative controller}

Figure 2 shows the time domain response of the hub $x_{h}$-motion and the first blade tip deflection $d_{1}$, calculated considering only the first bending mode, for the open-loop system (dashed-line) and the closed-loop system (solid-line) with the proportional derivative controller, developed in Sec. 3.5.
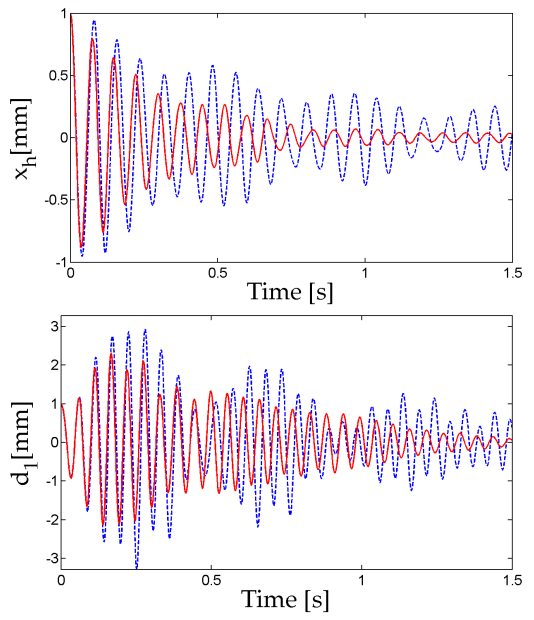

Figure 2: Hub displacements $x_{h}$ and the first blade tip deflection $d_{1}$.

It can be concluded that the PD controller can attenuate the vibration of the hub since the displacement $x_{h}$ decreases relatively fast. On the other hand, the PD controller has little effect on the blade deflection $d_{1}$.

\subsection{Single frozen model controller}

Figure 3 shows the response of the open-loop system (dashed-line) and the closed-loop system (solid-line) with the LQR controller derived using the single frozen model method from Sec. 3.3. The weighting matrices for the LQR design was chosen via a trial and error approach. The weighting matrix $W_{Q}$ was selected as a diagonal matrix with entries equal to $10^{7}$ for the hub related modes and equal to $10^{6}$ for modes related to the blades. The weighting matrix $W_{R}$ was also select as a diagonal matrix given by $W_{R}=10^{-1} I$.
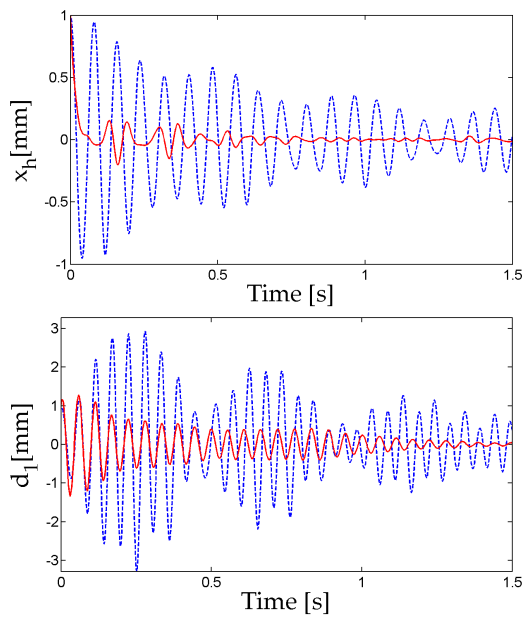

Figure 3: Hub displacement $x_{h}$ and of the first blade tip deflection $d_{1}$.

The effect of this controller is far better than the effect of the previous PD controller. The hub motion is suppressed effectively at the initial stage, however, when a certain level is reached the motion decays very slow. The suppression of the blade motion is not as effective as the one obtained for the hub motion, but it is still better than the system with the PD controller.

\subsection{Multiple frozen models controller}

Figure 4 shows the time domain response of the hub $x_{h}$-motion and the first blade tip deflection $d_{1}$ for the open-loop system (dashed-line) and the closed-loop system (solid-line) with the multiple frozen models controller from Sec. 3.4.

The weighting matrices $W_{Q}$ and $W_{R}$ have been chosen as the same as for the single frozen model controller. The time responses of the closed-loop system using this controller are seen to be quite similar to the frozen model controller. The blade motion decays quicker, however, after a minimum level has been reached, there is a remaining undesired blade vibration.

\subsection{Periodic LQR controller}

Figure 5 presents the results of the open-loop system (dashed-line) and the closed-loop system (solid-line) using the periodic LQR controller from Sect. 3.1. Trough numerical simulations, the 

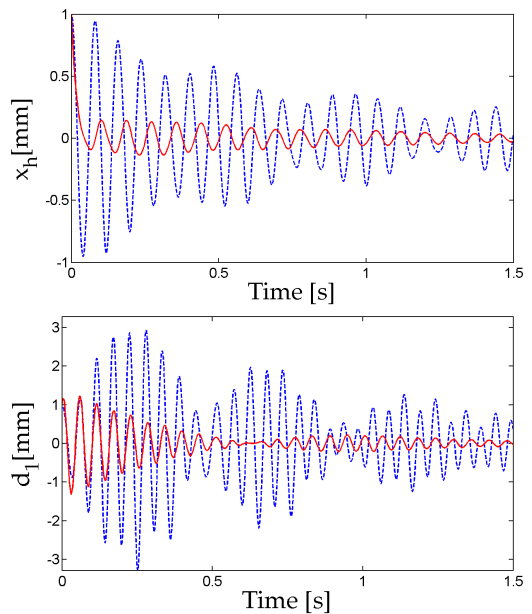

Figure 4: Hub displacement $x_{h}$ and of the first blade tip deflection $d_{1}$.

weighting matrix $W_{Q}$ has been chosen as a diagonal matrix with entries given by $10^{4}$ for the hub related modes and $10^{3}$ for the blade related modes. Note that since the states of the system (5) are decoupled, the matrix $Q$ can be chosen as diagonal. The control weighting matrix has been chosen as $W_{R}=10^{-1} I$.
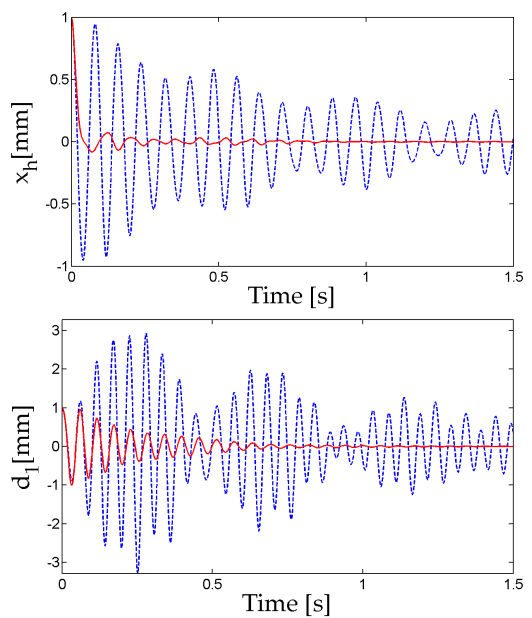

Figure 5: Hub displacement $x_{h}$ and of the first blade tip deflection $d_{1}$.

The periodic LQR controller improved significantly the performance of the closed-loop system compared to the previous controllers. This was expected, since this controller is guarantee to minimize a quadratic cost function that penalize the system states of the periodic system. The responses are seen to decay rapidly. Moreover, the vibrations of all the states die out completely, which were not the case with the previous controllers. It could have been hoped that the controller did not need as many periods of the blades vibrations to significantly reduce the blade deflection amplitudes. However, this controller is by far the best solution, considering its better perfor- mance and the fact it is able to ensure asymptotic stability of the closed-loop system.

\subsection{Feedforward controller}

In the previous sections, the unbalance was not included in the numerical simulations. Thus, the effects of the unbalance is now evaluated, together with the feedforward compensator, which was designed to cope with this known disturbance. The system has been simulated using the same initial condition as in the previous sections. Figure 6 shows the time response of the hub in the $\mathrm{x}$-direction and of the first blade tip deflection for the open-loop system without the unbalance (dashed-line), the unbalanced open-loop system (solid-line), and the unbalanced open-loop system with the feedforward controller (circle-line).
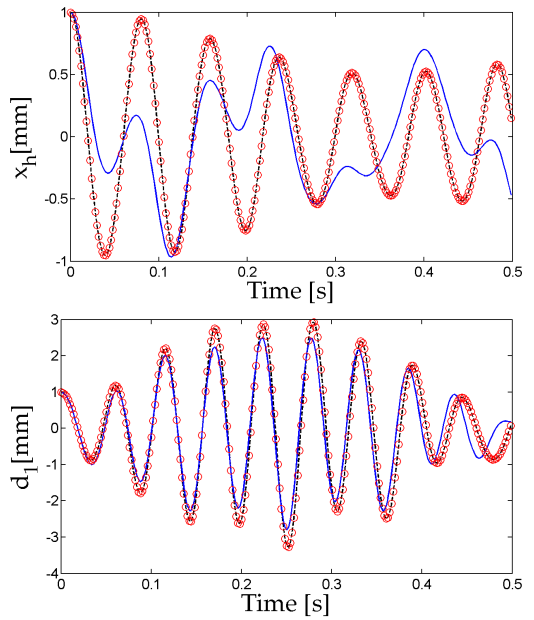

Figure 6: Hub displacement $x_{h}$ and of the first blade tip deflection $d_{1}$.

The effect of the of the unbalance is very evident, especially, in the hub response, giving a far different response. However, the implemented feedforward controller is able to completely cancel out the unbalance, giving a perfect match to the response of the unbalanced system. This was predicted in Sec. 3.6, meaning, that the pseudoinverse used is indeed effective.

\section{Conclusion}

The paper has proposed a technique to design a controller to attenuate the vibration of coupled rotor-blade systems via shaft actuation. An analytical model describing the dynamics of the rotor-blade system has been presented. A periodic modal decomposition method has been applied to decouple the system matrix. Four controllers have been implemented: a proportional derivative controller; an LQR controller based on a single frozen model; an LQR controller based on multiple frozen models; and a periodic LQR controller, which is the main contribution of this paper. The 
performance of the designed controllers have been verified through numerical simulations. The periodic LQR controller shows a superior performance compared to the others controllers. Moreover, the proposed periodic LQR controller is able to guarantee closed-loop stability.

\section{Acknowledgments}

This work is partially supported by the Brazilian funding agencies CAPES, CNPq, and FAPESP.

\section{References}

Anderson, B. D. O. and Moore, J. B. (1990). Optimal Control - Linear Quadratic Methods, PrenticeHall, Upper Saddle River, NJ, USA.

Arcara, P., Bittanti, S. and Lovera, M. (2000). Periodic control of helicopter rotors for attenuation of vibrations in forward flight, IEEE Transactions on Control Systems Technology 8(6): 883-894. DOI: $10.1109 / 87.880590$

Athans, M. (1971). The role and use of the stochastic linear-quadratic-gaussian problem in control system design, IEEE Transactions on Automatic Control 16(6): 529-552.

DOI: 10.1109/TAC.1971.1099818

Balas, M. J. (1978). Modal control of certain flexible dynamic systems, SIAM Journal of Control and Optimization 16(3): 450-462. DOI: $10.1137 / 0316030$

Baz, A. and Ro, J. (2001). Vibration control of rotating beams with active constrained layer damping, Smart Materials and Structures 10: 112-120. DOI: 10.1088/0964-1726/10/1/311

Boyd, S., El Ghaoui, L., Feron, E. and Balakrishnan, V. (1994). Linear Matrix Inequalities in Systems and Control Theory, Vol. 15 of Studies in Applied Mathematics, SIAM, Philadelphia.

Christensen, R. H. (2004). Active Vibration Control of Rotor-Blade Systems, Theory and Experiment, $\mathrm{PhD}$ thesis, Dept. of Mechanical Engineering, Technical University of Denmark, Kgs. Lyngby, Denmark.

Christensen, R. H. and Santos, I. F. (2005a). Design of active controlled rotor-blade systems based on time-variant modal analysis, Journal of Sound and Vibration 280: 863-882. DOI: 10.1016/j.jsv.2003.12.046

Christensen, R. H. and Santos, I. F. (2005b). Design of active controlled rotor-blade systems based on time-variant modal analysis, Journal of Sound and Vibration 280(3): 863-882. DOI: $10.1016 /$ j.jsv.2003.12.046

Christensen, R. H. and Santos, I. F. (2006). Active rotor-blade vibration control using shaftbased electromagnetic actuation, ASME Journal of Engineering for Gas Turbines and Powerd 128(3): 644-653. DOI: 10.1115/1.2056533

Firoozian, R. and Stanway, R. (1988). Active vibration control of turbomachinery: a numerical investigation of modal controllers, Mechanical Sys-tems and Signal Processing 2(3): 243-264. DOI: 10.1016/0888-3270(88)90022-2
Gusev, S. V., Shiriaev, A. S. and Freidovich, L. B. (2007). LMI approach for solving periodic matrix Riccati equation, Proceedings of the 3rd IFAC workshop on Periodic Control Systems, Anichkov Palace, Russia, pp. 254-256.

Hench, J. J. and Laub, A. J. (1994). Numerical solution of the discrete-time periodic Riccati equation, IEEE Transactions on Automatic Control 39(6): 1197-1210. DOI: 10.1109/9.293179

Johansson, S., Kaagstroem, B., Shiriaev, A. and Varga, A. (2007). Comparing one-shot and multishot methods for solving periodic Riccati differential equations, Proceedings of the 3rd IFAC workshop on Periodic Control Systems, Anichkov Palace, Russia, pp. 163-168.

Khulief, Y. A. (2001). Vibration suppression in rotating beams using active modal control, Journal of Sound and Vibration 242(4): 681-699. DOI: 10.1006/jsvi.2000.3385

Löfberg, J. (2004). YALMIP: A toolbox for modeling and optimization in MATLAB, Proc. 2004 IEEE Int. Symp. on Comput. Aided Control Syst. Des., Taipei, Taiwan, pp. 284-289.

Moore, J. B. and Anderson, B. D. . (1968a). Extensions of quadratic minimization theory I. Finite time results, International Journal of Con-trol 7(5): 465-472.

DOI: $10.1080 / 00207176808905631$

Moore, J. B. and Anderson, B. D. . (1968b). Extensions of quadratic minimization theory II. Infinite time results, International Journal of Control 7(5): 473-480. DOI: $10.1080 / 00207176808905632$

Sinha, S. C. and Joseph, P. (1994a). Control of general dynamic systems with periodically varying parameters via liapunov-floquet transformation, Journal of Dynamic Systems, Measurement and Control 116: 650-658. DOI: 10.1115/1.2899264

Sinha, S. C. and Joseph, P. (1994b). Control of general dynamic systems with periodically varying parameters via Liapunov-Floquet transformation, ASME Journal of Dynamic Systems, Measurement, and Control 116(4): 650-658.

DOI: $10.1115 / 1.2899264$

Sturm, J. F. (1999). Using SeDuMi 1.02, A MatLab toolbox for optimization over symmetric cones, Optimization Methods and Software 11(1): 625653. DOI: $10.1080 / 10556789908805766$

Szász, G. and Flowers, G. T. (2001). Time periodic control of a bladed disk assembly using shaft based actuation, ASME Journal of Vibration and Acoustics 123(3): 395-398. DOI:10.1115/1.1375163

Varga, A. (2005). On solving periodic differential matrix equations with applications to periodic system norms computation, Proceedings of the 44th IEEE Conference on Decision and Control - European Control Conference 2005, Seville, Spain, pp. 6545-6550. DOI:10.1109/CDC.2005.1583212

Willems, J. C. and Mitter, S. K. (1971). Controllability, observability, pole allocation, and state reconstruction, IEEE Transactions on Automatic Control 16(6): 582-595. DOI: $10.1109 /$ TAC.1971.1099819

$\mathrm{Xu}, \mathrm{J}$. and Gasch, R. (1995). Modale behandlung linearer periodisch zeitvarianter bewegungsgleichungen, Archive of Applied Mechanics 65(3): 178-193. DOI: $10.1007 / \mathrm{s} 004190050011$ 